\title{
Extracellular matrix differences in glioblastoma patients with different prognoses
}

\author{
JÓZSEF VIRGA $^{1 *}$, LÁSZLÓ SZIVOS ${ }^{1 *}$, TIBOR HORTOBÁGYI ${ }^{2}$, MAHAN KOUHSARI CHALSARAEI $^{2}$, \\ GÁBOR ZAHUCZKY ${ }^{3}$, LÁSZLÓ STEINER ${ }^{3}$, JUDIT TÓTH ${ }^{4}$, JUDIT REMÉNYI-PUSKÁR ${ }^{1}$, \\ LÁSZLÓ BOGNÁR ${ }^{1 * *}$ and ALMOS KLEKNER ${ }^{1 * *}$ \\ ${ }^{1}$ Department of Neurosurgery, Faculty of Medicine, University of Debrecen; \\ ${ }^{2}$ MTA-DE Cerebrovascular and Neurodegenerative Research Group, Department of Neuropathology, \\ Institute of Pathology, University of Debrecen; ${ }^{3}$ UD Genomed Ltd.; ${ }^{4}$ Department of Oncology, \\ Faculty of Medicine, University of Debrecen, H-4032 Debrecen, Hungary
}

Received April 11, 2018; Accepted August 24, 2018

DOI: $10.3892 / \mathrm{ol} .2018 .9649$

\begin{abstract}
Glioblastoma is the most common malignant central nervous system tumor. Patient outcome remains poor despite the development of therapy and increased understanding of the disease in the past decades. Glioma cells invade the peritumoral brain, which results in inevitable tumor recurrence. Previous studies have demonstrated that the extracellular matrix (ECM) is altered in gliomas and serves a major role in glioma invasion. The present study focuses on differences in the ECM composition of tumors in patients with poor and improved prognosis. The mRNA and protein expression of 16 invasion-associated ECM molecules was determined using reverse trascription-quantitiative polymerase chain reaction and immunohistochemistry, respectively. Clinical factors of patients with different prognoses was also analyzed. It was determined that age and postoperative Karnofsky performance score were associated with patient survival. Furthermore, Fms-related tyrosine kinase 4/vascular endothelial growth factor receptor 3 (FLT4/VEGFR3), murine double minute 2 (MDM2) and matrix metallopeptidase 2 (MMP2) mRNA levels were significantly different between the two prognostic groups. Additionally, brevican, cluster of differentiation 44, hyaluronan mediated motility receptor, integrin- $\alpha \mathrm{V}$ and $-\beta 1$, and MDM2 protein expression were indicated to be significantly different in immunohistochemistry slides. Using the expression profile, including the invasion spectrum of the samples, it was possible to identify the prognostic group of the sample with high efficacy, particularly in cases with
\end{abstract}

Correspondence to: Dr Almos Klekner, Department of Neurosurgery, Faculty of Medicine, University of Debrecen, 98 Nagyerdei krt., H-4032 Debrecen, Hungary

E-mail: neurosurgery.debrecen@freemail.hu

\section{${ }^{* * * *}$ Contributed equally}

Key words: glioblastoma, extracellular matrix, survival, expression, prognosis poor prognosis. In conclusion, it was determined that ECM components exhibit different expression levels in tumors with different prognoses and thus the invasion spectrum can be used as a prognostic factor in glioblastoma.

\section{Introduction}

Glioblastoma is a disease with a notably poor prognosis, with a 2 -year prognosis is as low as $27 \%$ in Europe and in the United States $(1,2)$. Patients undergo concurrent chemoradiation following tumor resection, and then chemotherapy, namely alkylating agent temozolomide, is administered as monotherapy. Currently, only bevacizumab, an anti-vascular endothelial growth factor (VEGF) antibody, is available as a targeted therapy (2-4). Despite the changes in treatment protocols in the past decade and the extensive research in the field of glioblastoma treatment options, the majority of patients succumb 16-24 months after diagnosis (1-4). Specific clinical factors affect patient outcome, including sex, age, Karnofsky performance status (KPS) score and tumor size, but these are poor predictors of prognosis and cannot be used successfully in everyday clinical practice $(5,6)$. Maximum safe resection may improve patient survival; however, neither the minimal cut-off resection volume nor the maximum residual tumor volume that is associated with survival benefit has been established (7).

The molecular pathology of glioblastomas has been the focus of recent research (8-13). Certain well-established alterations in tumors, including methylation of the O-6-methylguanine-DNA methyltransferase (MGMT) promoter region, isocitrate dehydrogenase (IDH) 1/2 or telomerase reverse transcriptase mutations, epidermal growth factor receptor (EGFR) amplification or the well-known variant III mutation, are also associated with prognosis (14). IDH mutations have a notable impact on patient outcome; however, they are present in $<10 \%$ of glioblastomas $(15,16)$. MGMT promoter methylation status is associated with the effectiveness of the current standardly used temozolomide chemotherapy, but alterations in the methylation status during treatment is a known phenomenon (9). Currently, clinicians have no means 
of assessing the prognosis of individual patients, even though there would be a demand for identifying patient subgroups with different survival chances. Identifying patients that require more frequent follow-up examinations to detect tumor recurrence and determining subgroups that would benefit less from the aggressive treatment protocol currently used, or that would be fit for targeted therapy, is currently not possible for the majority of clinicians.

Peritumoral infiltration is a hallmark of all diffusely growing glioma types, therefore glioblastomas are also invasive (10). Tumor masses have no clear macroscopic border, thus complete surgical resection is not possible (7). The extracellular matrix (ECM) is known to be an important determinant of glioma invasion. Previous studies revealed alterations in the expression of specific ECM components in glioblastoma compared with that in non-tumor brain or grade II-III astrocytomas (17-20). Certain inhibitors targeted against specific ECM components have been previously tested in clinical trials, but have failed to provide a breakthrough in glioblastoma treatment thus far (1,4,21-23). A selected group of ECM-associated molecules, also known as an invasion panel, was tested in previous studies to understand the molecular behavior of glioblastoma. The expression pattern of these molecules, including the invasion spectrum, was demonstrated to be specific for primary and secondary tumors, as well as the grade of astrocytomas (24-26). Furthermore, it is considered that the molecular composition of glioblastoma ECM contributes to invasiveness; thus, the molecular fingerprint of the tumor is associated with the efficacy of conventional and targeted therapies. For example, matrix metallopeptidase 2 (MMP2) and MMP9 molecules are associated with increased invasiveness in glioblastoma. Integrin- $\alpha \mathrm{V}$ (ITGAV) is known to be involved in the migration of glioma cells and is thus considered to contribute to invasiveness. Other examples include the hyaluronan receptor cluster of differentiation (CD)44, or the central nervous system-specific proteoglycan brevican (BCAN), which have been associated with increased invasiveness $(24,27-29)$. The aim of the present study was to analyze the expression pattern of the invasion panel in glioblastoma samples, and to identify the association between the invasion spectrum and disease progression.

\section{Patients and methods}

Patients and tumor samples. Samples from 132 adult (58 females and 74 males) patients who were diagnosed with primary glioblastoma between 01 January 2006 and 31 December 2015 were selected from the Brain Tumor and Tissue Bank of the Department of Neurosurgery, University of Debrecen (Debrecen, Hungary). Stratified random sampling was performed, with overall survival time as stratum. No other parameter was considered when selecting samples. The tumor samples were all obtained from brain lobes, and were frozen intraoperatively on the surface of liquid nitrogen and then stored at $-78^{\circ} \mathrm{C}$ in a deep freezer until use for mRNA analysis. The rest of the resected tumor was fixed in $10 \%$ paraformaldehyde solution at $25^{\circ} \mathrm{C}$ for $24 \mathrm{~h}$ and then embedded in paraffin. The tissues were examined, and the diagnosis of glioblastoma was made by an experienced neuropathologist. Patients had been treated
Table I. List of the analyzed ECM components.

\begin{tabular}{ll}
\hline Symbol & \multicolumn{1}{c}{ Assay ID } \\
\hline BCAN & BCAN-Hs00222607_m1 \\
CD44 & CD44-Hs01075861_m1 \\
CSPG5 & CSPG5-Hs00198108_m1 \\
EGFR & EGFR-Hs01076090_m1 \\
FLT4/VEGF3 & FLT4-Hs00176607_m1 \\
HMMR & HMMR-Hs00234864_m1 \\
IDH1 & IDH1-Hs01855675_s1 \\
ITGAV & ITGAV-Hs00233808_m1 \\
ITGB1 & ITGB1-Hs00559595_m1 \\
ITGB5 & ITGB5-Hs00174435_m1 \\
MDM2 & MDM2-Hs01066930_m1 \\
MMP-2 & MMP2-Hs01548727_m1 \\
NCAN & NCAN-Hs00189270_m1 \\
PDGFA & PDGFA-Hs00964426_m1 \\
TNC & TNC-Hs01115665_m1 \\
VCAN & VCAN-Hs00171642_m1 \\
GFAP & GFAP-Hs00909233_m1 \\
MKI67 & MKI67-Hs01032443_m1 \\
B2M & B2M-Hs00187842_m1 \\
GAPDH & GAPDH-Hs99999905_m1
\end{tabular}

A total of 16 invasion-associated ECM molecules were selected for Taqman Low Density Arrays. GFAP and MKI67 were used for quality control of the samples. B2M and GAPDH were used as housekeeping genes, and $\mathrm{B} 2 \mathrm{M}$ was selected as the reference gene for relative gene expression analyses. ECM, extracellular matrix; BCAN, brevican; CD44, cluster of differentiation 44; CSPG5, chondroitin sulfate proteoglycan 5; EGFR, epidermal growth factor receptor; FLT4, Fms-related tyrosine kinase 4; HMMR, hyaluronan-mediated motility receptor; IDH1, isocitrate dehydrogenase 1; ITGAV, integrin- $\alpha \mathrm{V}$; MDM2, murine double minute 2; MMP-2, matrix metallopeptidase 2; NCAN, neurocan; PDGFA, platelet-derived growth factor $\alpha$; TNC, tenascin C; VCAN, versican; VEGF, vascular endothelial growth factor; GFAP, glial acidic fibrillary protein; MKI67, marker of proliferation Ki-67; B2M, $\beta 2$ microglobulin.

with radiotherapy plus concomitant and maintenance temozolomide chemotherapy following maximal safe resection of the tumor. Upon tumor recurrence, patients received bevacizumab monotherapy until further progression. Patient age ranged from 20 to 82 years, median age was 60 years [confidence interval (CI), 58.0-62.0 years], and the mean postoperative KPS score was 82.5 (CI, 80.4-84.7). Overall mean survival (OS) time of the patients was 20.8 months (CI, 17.6-24.0 months). Patients were divided into two prognostic groups based on OS time. Patients whose OS time was $<24$ months were considered to be "patients with a worse prognosis' and were placed in group A $(n=74)$, whereas patients whose OS time was $\geq 24$ months were considered to be 'patients with a better prognosis' and were placed in group B $(n=58)$. The 24-month threshold was selected as a previously published analysis of patient data at our institution (University of Debrecen Clinical Center, Department of Neurosurgery, Department of 
Table II. Primary antibodies used for immunohistochemical staining.

\begin{tabular}{lllrl}
\hline Protein & \multicolumn{1}{c}{ Antibody code } & \multicolumn{1}{c}{ Manufacturer } & Dilution & Positive control \\
\hline Brevican & NBP1-89992PEP & Novus Biologicals (Littleton, CO, USA) & $1: 200$ & Rat brain \\
CD 44 & AB16728 & Abcam (Cambridge, United Kingdom) & $1: 500$ & Human tonsil \\
CSPG-5 (neuroglyan C) & ORB157961 & Biorbyt (Cambridge, United Kingdom) & $1: 250$ & Rat brain \\
Flt-4/VEGFR-3 & SC-514825 & Santa Cruz Biotechnology (Dallas, TX, USA) & $1: 250$ & Human kiney \\
HMMR (CD168, RHAMM) & AB110075 & Abcam (Cambridge, United Kingdom) & $1: 200$ & Human tonsil \\
Integrin alpha V chain & BS-2203R & Bioss Antibodies (Woburn, MS, USA) & $1: 250$ & Human kidney \\
Integrin beta 1 chain & RD-MAB1778-SP & RnD Systems (Minneapolis MN, USA & $1: 250$ & Human tonsil \\
Integrin beta 5 chain & NBP1-88117 & Novus Biologicals (Littleton, CO, USA) & $1: 250$ & Human tonsil \\
MDM2 & AB16895 & Abcam(Cambridge, United Kingdom) & $1: 400$ & Rat brain \\
MMP-2 & TA806846 & OriGene Technologies (Rockville, MD, USA) & $1: 200$ & Human tonsil \\
Versican & AB177480 & Abcam (Cambridge, United Kingdom) & $1: 150$ & Rat brain \\
\hline
\end{tabular}

Oncology, Debrecen, Hungary) determined that patients previously treated according to Stupp's protocol (60 Gy irradiation with concurring $75 \mathrm{mg} / \mathrm{d} / \mathrm{m}^{2}$ temozolomide, followed by $150-200 \mathrm{mg} / \mathrm{d} / \mathrm{m}^{2}$ temozolomide monotherapy until progression), then receiving bevacizumab following disease progression have a mean OS time of 24 months (24). Literature data also supported that an OS of $\geq 24$ months can be considered to represent better than average survival $(5,30)$.

Determination of the invasion spectrum. The invasion spectrum was determined by measuring the mRNA expression level of the 16 molecules of the invasion panel. Ki-67 was measured to confirm presence of tumor tissue, GFAP was measured to confirm glial origin. (Table I). The mRNA expression was determined through reverse transcriptase-quantitative polymerase chain reaction (RT-qPCR), as described previously $(31,32)$. Flash-frozen tissue samples were first pulverized and then homogenized using TriReagent ${ }^{\circledR}$ (Invitrogen; Thermo Fisher Scientific, Inc., Waltham, MA, USA) at $25^{\circ} \mathrm{C}$ until no visible solid tissue was present in the tubes. Total RNA was isolated from TriReagent lysates, according to the manufacturer's protocols. A NanoDrop ${ }^{\circledR}$ ND-1000 Spectrophotometer (NanoDrop Technologies; Thermo Fisher Scientific, Inc., Wilmington, DE, USA) was used to measure the quantity and purity of the RNA. Subsequently, reverse transcription was performed to convert total RNA to single-stranded complementary DNA (cDNA) with a High-Capacity cDNA Archive kit with RNasin (Applied Biosystems; Thermo Fisher Scientific, Inc.). The cDNA was then loaded onto a microfluidic card (cDNA from 100 ng of total RNA/port). An Applied Biosystems 7900HT real-time PCR system with Micro Fluidic Card upgrade (Applied Biosystems; Thermo Fisher Scientific, Inc.) was used to perform TaqMan ${ }^{\circledR}$ low-density array experiments. The thermocycling conditions were $2 \mathrm{~min}$ at $50^{\circ} \mathrm{C}$, $10 \mathrm{~min}$ at $94.5^{\circ} \mathrm{C}$, followed by 40 cycles of denaturation at $97^{\circ} \mathrm{C}$ for $30 \mathrm{sec}$ and annealing and extension at $60^{\circ} \mathrm{C}$ for $1 \mathrm{~min}$. The Micro Fluidic Cards were analyzed with SDS 2.1 software (Applied Biosystems; Thermo Fisher Scientific, Inc.) as relative quantification studies, and the cycle quantification $(\mathrm{Cq})$ values were exported for further analysis. $\beta 2$ microglobulin and GAPDH housekeeping genes exhibited the least variation among the samples, and $\beta 2$ microglobulin was used as reference gene to calculate the $\Delta \mathrm{Cq}$ value for each gene. Expression values were calculated using the comparative $\mathrm{Cq}$ method, as described previously (33).

Immunohistochemistry. Protein expression was measured by immunohistochemical staining of formalin-fixed paraffin embedded tissue slides. For the slides, the corresponding tissue blocks were collected from the Department of Neuropathology, Institute of Pathology, University of Debrecen. Sections $(4 \mu \mathrm{m})$ were cut with a microtome then prepared for staining. Sections were first deparaffinized using xylene, then rehydrated in a graded series $(100 \%$, then $95 \%$ ) of ethanol at $25^{\circ} \mathrm{C}$. Epitopes were retrieved by heat-induction in citrate buffer $(\mathrm{pH}$ 6.0, Antigen Decloaker 10X, Biocare Medical, LLC, Pacheco, CA, USA). Endogenous peroxidase activity was blocked with $0.3 \%$ methanolic hydrogen-peroxide at $25^{\circ} \mathrm{C}$ for $10 \mathrm{~min}$ prior to the slides being incubated overnight with the primary antibodies in concentrations according to manufacturer's protocols. (Table II) Visualization was conducted with a MACH 4 Universal AP Polymer kit (Biocare Medical, LLC, Paheco, CA, USA) according to manufacturer's protocol and 3,3'-diaminobenzidine with hematoxylin counterstaining at $25^{\circ} \mathrm{C}$ for $10 \mathrm{~min}$ and $30 \mathrm{secs}$, respectively. Positive and negative control slides were also stained using the same procedures as the other slides (Table II). Expression of the proteins was evaluated by three independent observers in 10 random high-power fields (x100 magnification, Eclipse $80 \mathrm{i}$ microscope, Nikon, Minato, Tokyo, Japan) in each slide in a semi-quantitative manner, considering the percentage of staining cells and/or extracellular space, as well as the intensity of staining. The percentage of the staining was considered as follows: 0 , negative or $<10 \%$ staining; 1 , $10-25 \%$ staining; $2,26-50 \%$ staining; $3,51-75 \%$ staining; and $4,>75 \%$ staining, as described by Bondarendko et al (34). Intensity was graded from 0-3 (negative, -; weak positivity, +; moderate positivity, ++ ; and strong positivity, +++ , respectively). Combined scores were calculated for each slide by multiplying the scores, and a mean score for each sample was then determined. 


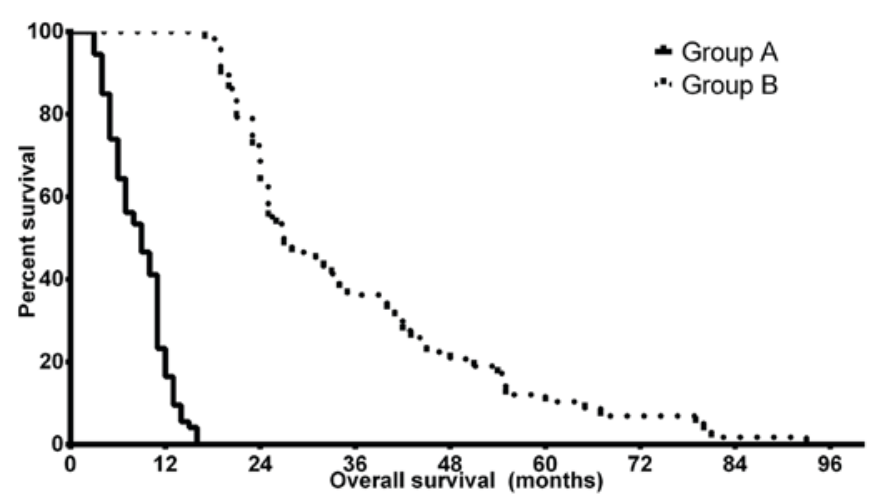

Figure 1. Kaplan-Meier survival analysis of patients with glioblastoma with different disease prognoses. Patients in group A had significantly reduced OS time compared with those in group B. Group A, OS $<24$ months; group B, OS $>24$ months; OS, overall survival.

Statistical analysis. Independent samples Student's paired $\mathrm{t}$-test and Mann-Whitney U tests were used for age, tumor size, KPS values, RNS and protein expression, while $\chi^{2}$ test was used for sex, tumor location and percentage of repeat surgery patients. Progression-free survival (PFS) and OS times were compared using Kaplan-Meier survival analysis with log-rank (Mantel-Cox) test. PFS and OS times were calculated from the time of diagnosis. For an overall analysis of the expressional pattern, the statistical classifier nearest neighbor search was used on mRNA expression data $(35,36)$. The nearest neighbor search analyzed all attributes (i.e. the level of expression of a given ECM molecule) at the same time and compared these attributes to those of other samples, finding similar patterns of mRNA expression among the samples. The value of every measured ECM component were used to identify samples that have the most similar values. Samples with similar patterns of expression were then put in the same group. $\mathrm{P}<0.05$ level of significance was considered as statistically significant. Calculations were performed using statistical program GraphPad Prism v6.01 (GraphPad Software Inc., La Jolla, CA, USA). Nearest neighbor search was performed using statistical program Weka v3.6 (University of Waikato, Hamilton, New Zealand).

\section{Results}

Patient data. The clinical characteristics of the patients are summarized in Table III. The median ages of the patients was 61.0 years (CI, 57.97-63.28 years) in group A and 58.5 years (CI, 55.53-61.47 years) in group B. Statistical analysis revealed a significant difference between the two groups $(\mathrm{P}=0.0293)$. Patients had an improved preoperative and postoperative KPS score in group B compared with patients in group A, but the KPS scores did not differ significantly. The other examined clinical characteristics of the tumors were not statistically different in the two groups: the tumors were determined to be similar in size in the two groups and there was no statistical difference in the involvement of the dominant side of the brain nor the lobular localization of the tumor. Kaplan-Meier analyses confirmed the predicted differences in PFS and OS of the patients in groups A and B. The median OS time was 9.0 months (CI, 8.14-9.86 months) in group A, while patients in group B had a significantly improved prognosis with a median 


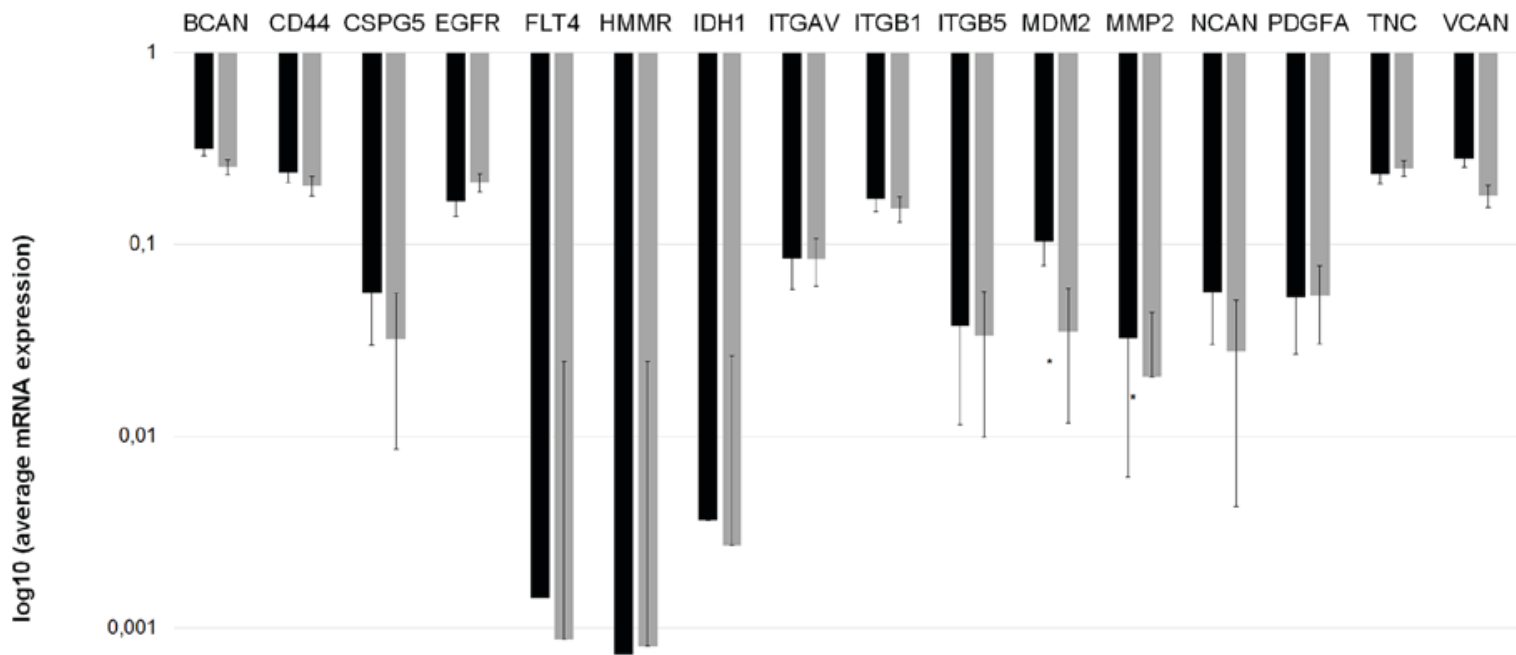

Figure 2. Invasion spectrum (the mRNA expression pattern of invasion-associated extracellular matrix components) differs in patients with 'worse' and 'better' prognoses. mRNA expression measurements were performed twice for each gene to confirm the data. A longer bar on the logarithmic scale indicates reduced expression. "P<0.05 vs. group A (Mann-Whitney U test). Group A, OS <24 months; group B, OS >24 months; BCAN, brevican; CD44, cluster of differentiation 44; CSPG5, chondroitin sulfate proteoglycan 5; EGFR, epidermal growth factor receptor; FLT4, Fms-related tyrosine kinase 4; HMMR, hyaluronan-mediated motility receptor; IDH1, isocitrate dehydrogenase 1; ITGAV, integrin- $\alpha \mathrm{V}$; MDM2, murine double minute 2; MMP-2, matrix metallopeptidase 2; NCAN, neurocan; PDGFA, platelet-derived growth factor $\alpha$; TNC, tenascin C; VCAN, versican; OS, overall survival.

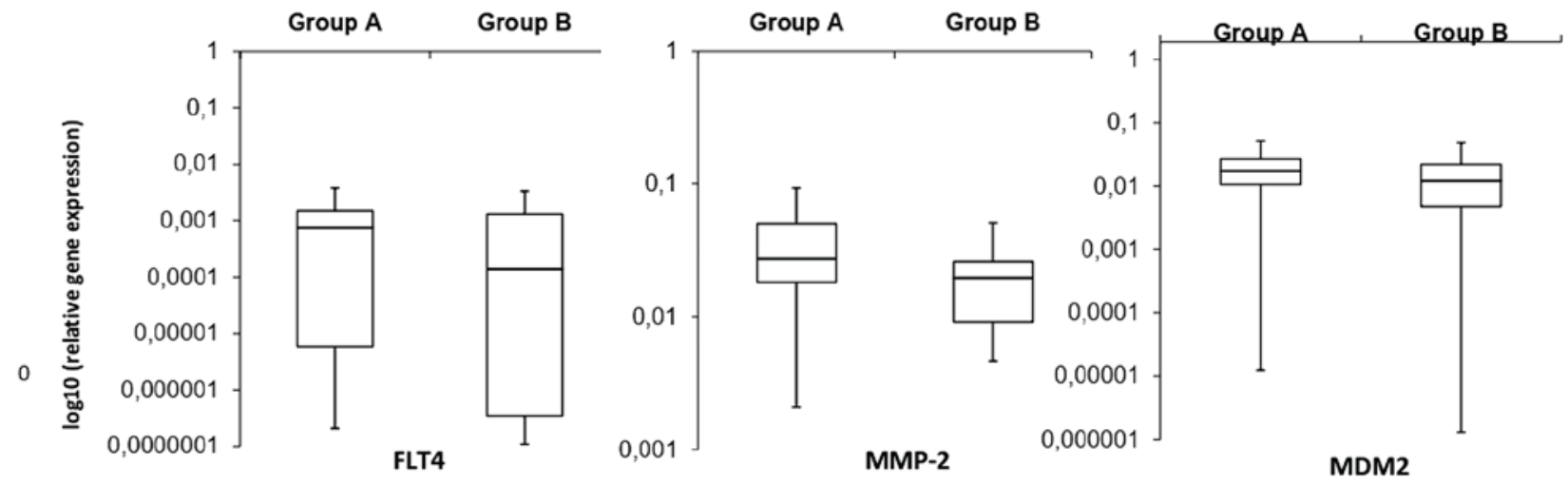

\begin{tabular}{|c|rc|cc|c|}
\hline Mean rel. expression & 0,001435 & 0,000876 & 0,032295 & 0,020476 & 0,076221 \\
\hline & & & & 0,035211 \\
\hline 25th percentile & 0,000006 & 0,000001 & 0,018163 & 0,009185 & 0,010584 \\
\hline 50th percentile (median) & 0,000737 & 0,000139 & 0,027470 & 0,019750 & 0,004792 \\
\hline 75th percentile & 0,001496 & 0,001310 & 0,050537 & 0,026029 & 0,017177 \\
\hline p-value & \multicolumn{2}{|c|}{0.0285} & \multicolumn{2}{|c|}{0.0200} & 0,027092 \\
\hline
\end{tabular}

Figure 3. A total of 3 invasion-associated extracellular matrix components demonstrated significantly different expression levels in the samples from different prognostic groups. All 3 molecules had increased expression in patients in group A, indicating that the level of these molecules was associated with tumor invasiveness and patient survival. Group A, OS <24 months; group B, OS > 24 month.; FLT4, Fms-related tyrosine kinase 4; MDM2, murine double minute 2; MMP-2, matrix metallopeptidase 2; rel., relative; OS, overall survival.

OS time of 27.0 months (CI, 22.17-31.83 months) $(\mathrm{P}<0.0001)$ The median PFS differences were less notable but remained statistically significant, with 5.5 months (CI, 3.79-7.21 months) in group A and 8.5 months (4.36-12.64 months) in group B $(\mathrm{P}=0.024)$. Fig. 1 depicts the OS differences between groups $\mathrm{A}$ and $\mathrm{B}$. The two groups therefore had no major differences in the clinical characteristics of the patients apart from patient age and the survival times, which were selection criteria.

mRNA expression of the tumor samples. Ki-67 mRNA was measured in each sample to confirm that the flash-frozen samples contained sufficient tumor tissue. All the samples were determined to express sufficient marker of proliferation Ki-67 (MKI67) and the two groups did not demonstrate any significant difference in Ki-67 expression ( $\mathrm{P}=0.8567)$. GFAP expression was also analyzed to confirm the presence of glial tumors.

The mRNA expression of the invasion-associated ECM components in the two prognostic groups demonstrated notable differences. Fig. 2 depicts the mean mRNA expression values of the analyzed molecules. Using the independent samples Student's t-test, 3 invasion-associated molecules demonstrated 
Table IV. Mean immunohistochemistry scores of the ECM components that were determined to be significantly different in the two prognostic groups.

Mean

immunohistochemistry scores

\begin{tabular}{lcccc}
\cline { 3 - 4 } ECM component & P-value & Concordance with mRNA results & Group A & Group B \\
\hline BCAN & 0.0002 & Yes & 6.58 & 3.40 \\
CD44 & 0.0200 & Yes & 4.35 & 3.69 \\
HMMR & 0.0020 & Yes & 5.71 & 3.58 \\
ITGAV & Yes & 4.35 & 3.69 \\
ITG $\beta 1$ & 0.0200 & No & 2.69 & 3.86 \\
MDM2 & 0.0400 & Yes & 4.41 & 2.44 \\
\hline
\end{tabular}

Group A, OS <24 months; group B, OS > 24 months; ECM, extracellular matrix; ITGAV, integrin- $\alpha \mathrm{V}$; BCAN, brevican; CD44, cluster of differentiation 44; HMMR, hyaluronan-mediated motility receptor; MDM2, murine double minute 2; OS, overall survival.

Table V. Characteristics of the binary classification that identified the prognostic group of each sample based upon the mRNA expression pattern of the extracellular matrix of the samples.

\begin{tabular}{lccccc}
\hline Class & Sensitivity & FP rate & Positive prediction value & F-1 score & MCC \\
\hline Group A & 0.757 & 0.345 & 0.737 & 0.747 & 0.414 \\
Group B & 0.655 & 0.243 & 0.679 & 0.667 & 0.4144 \\
Weighted mean & 0.712 & 0.300 & 0.711 & 0.712 & 0.414 \\
\hline
\end{tabular}

Group A, OS <24 months; group B, OS > 24 months; ROC, receiver operating curve; OS, overall survival; FP, false positive; F-1, F function score; MCC, Matthew's correlation coefficient.

significant differences between the two prognostic groups. Fms-related tyrosine kinase 4 (FLT4), murine double minute 2 (MDM2) and MMP2 expression levels were significantly increased in patients in group A compared with those in group $\mathrm{B}(\mathrm{P}=0.0285, \mathrm{P}=0.0200$ and $\mathrm{P}=0.0023$, respectively). Fig. 3 depicts the expression of these 3 ECM components in the different prognostic groups.

Immunohistochemistry confirms different ECM composition in tumors with different prognoses. Immunohistochemistry sections were analyzed and differences in protein expression were identified in the different glioblastoma prognostic groups. Out of the analyzed invasion-associated proteins, BCAN $(\mathrm{P}=0.0002), \mathrm{CD} 44(\mathrm{P}=0.0200)$, hyaline-mediated motility receptor $(\mathrm{P}=0.0020)$, ITGAV and $-\beta 1(\mathrm{P}=0.0200$ and 0.0400 , respectively), and MDM2 $(\mathrm{P}=0.0240)$ were determined to be significantly different between the two groups. The mRNA and immunohistochemical differences were concordant in direction except for ITG $\beta 1$ molecules. Concordance of protein expression underlines the relevance of the mRNA expression data. Table IV contains the immunohistochemistry scores and P-values of the significant ECM molecules. Immunohistochemical slides of the ECM components that were significant at the mRNA level are presented in Figs. 4-6. with worse and better prognoses. Subsequent to analyzing
mRNA and protein expression for each ECM component individually, a nearest neighbor search, a statistical classifier to perform joint, overall analysis of the invasion panel, was used. The following molecules were used by the statistical classifier algorithm as major influencers: CD44, EGFR, FLT4/VEGF-3, IDH1, MMP2, platelet-derived growth factor $\alpha$, tenascin $\mathrm{C}$ and versican.

The expression of these ECM components may be a key component in the separation of tumors with different prognoses. The classifier identified the prognosis, including whether the patient lived $\geq 24$ months, in $94 / 132$ patients correctly. Sensitivity and positive predictive values were increased in tumors with a worse prognosis. The method's receiver operating curve (ROC) area, which is the curve of sensitivity dotted against false positive rate, is 0.706 . As the value rages from 0 to 1 , a value of 0.706 suggests good accuracy. The Matthew's correlation coefficient is 0.414 . MCC measures the quality of binary classification and its values range from -1 to +1 , where 0 is no better than random classification and +1 is perfect classification, thus a coefficient of 0.414 indicates fine selection between the groups. Further details of the method are within Table V.

\section{Discussion}

Glioblastoma, the most common glioma, has a poor prognosis $(1,2)$. Despite aggressive treatment protocols, patients 


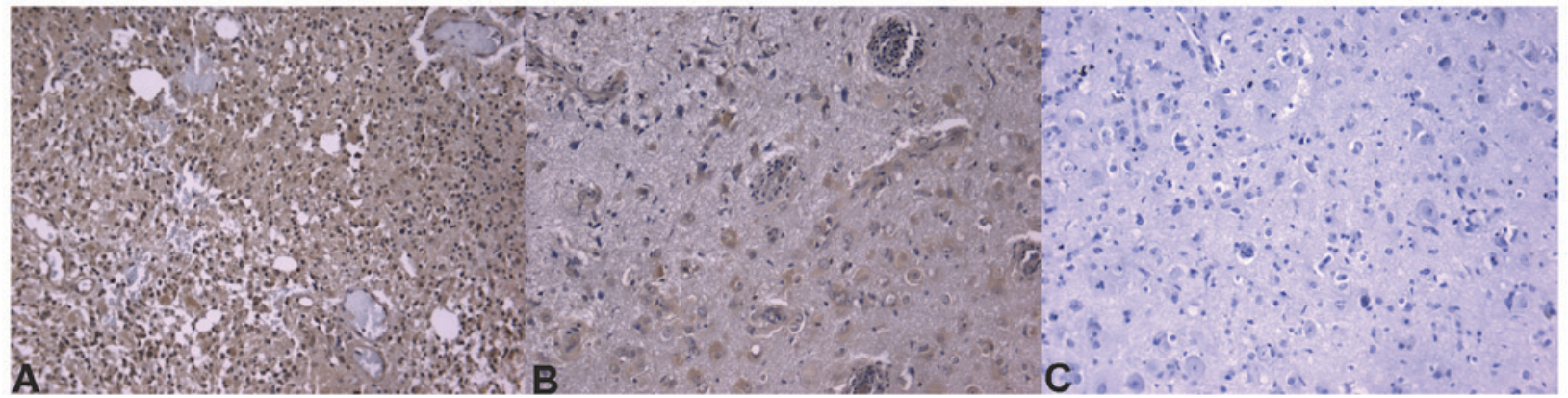

Figure 4. Immunohistochemical images of glioblastoma stained for FLT4/VEGF-3. (A) Overexpression of FLT4/VEGF-3 in patients with glioblastoma with a worse prognosis. (B) Moderate positivity in patients with a better prognosis (staining is notably reduced compared with that in patients with a worse prognosis). (C) Negative control. All images depicted are at x20 magnification. FLT4, Fms-related tyrosine kinase 4; VEGF, vascular endothelial growth factor.

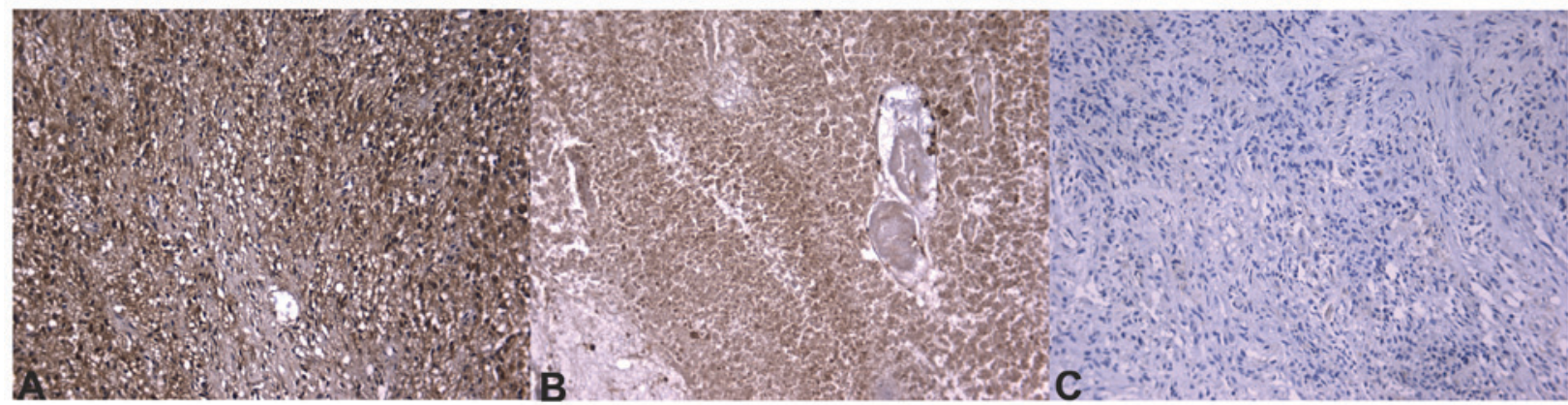

Figure 5. Immunohistochemical images of glioblastoma stained for MDM2. (A) Immunhistochemical images from patients with a worse prognosis demonstrated significantly increased positivity for MDM2, compared with (B) those with a better prognosis. (C) Negative control. All images are depicted at x20 magnification. MDM2, murine double minute 2 .

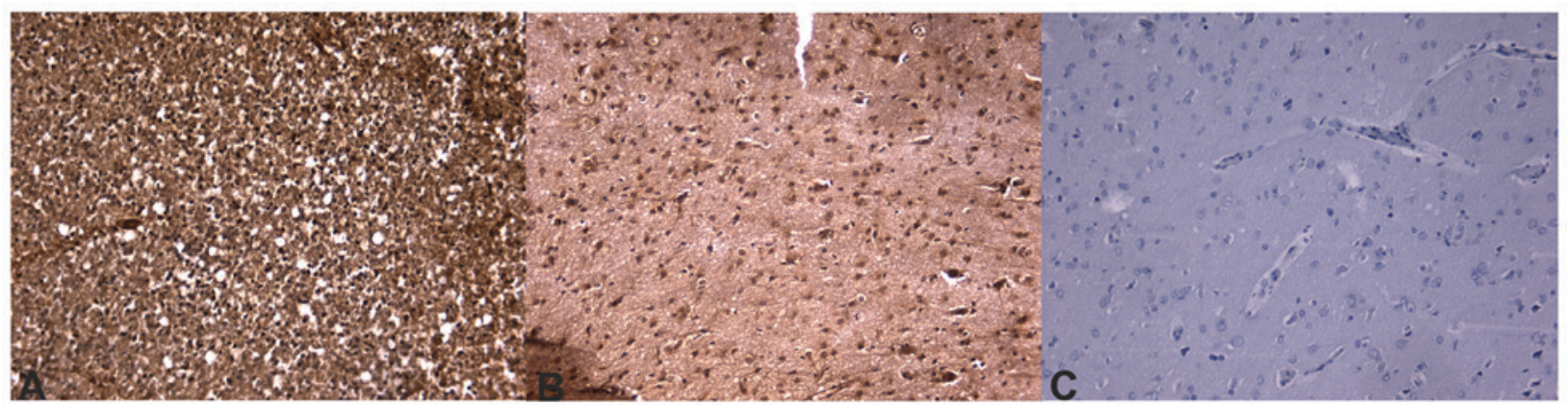

Figure 6. Immunohistochemical images of glioblastoma stained for MMP-2. (A) MMP-2 positivity was notably strong in immunohistochemical images from patients with a worse prognosis, confirming the mRNA expression results. (B) Patients with a better prognosis expressed an increased amount of MMP-2, as the strong positivity indicates, but the immunohistochemical scores are significantly reduced. (C) Negative control slide. All images are depicted at x20 magnification. MMP-2, matrix metallopeptidase 2.

survive for only 16-24 months following diagnosis $(37,38)$. Certain factors, like age or maximal safe tumor resection, are known to have a degree of association with patient survival; however, clinicians cannot assess individual prognosis (5-7). Glioma cells invade the neighboring tissue in a complex process actively influenced by ECM components $(28,39)$. Previous studies determined that certain ECM molecules were expressed at an altered, frequently increased level in gliomas compared with that in non-tumor brain samples, and numerous studies determined that certain drugs designed to target these molecules can inhibit tumor invasion in vitro in certain subgroups of patients with glioblastoma $(3,24,40-45)$. Bevacizumab is highly effective in one subgroup of patients, while other patients have reduced or no benefit from the targeted therapy $(3,46,47)$. Furthermore, bevacizumab has been determined to exert its effect primarily in increasing PFS time, and it may not affect OS time significantly $(48,49)$.

The present study aimed to analyze the ECM composition of glioblastoma samples in order to identify the expression pattern of patients with glioblastoma with 'worse' or 'better' survival. The two prognostic groups demonstrated differences in patient age at diagnosis, which is in accordance with literature data $(5,6)$. No further differences were determined in terms of KPS score, tumor size or tumor location; therefore, it is possible that differences in survival are not explained by differences in clinical factors, as all patients had undergone the identical treatment 
policy; however, the survival times were notably different. Molecular methods were used to investigate differences in the molecular composition of the tumors with different prognoses.

It was determined that ECM components demonstrate differences in expression at transcriptional or translational levels. The mRNA expression of FLT4, MDM2 and MMP2 genes was determined to be significantly different between the two groups. Additionally, MDM2 expression was determined to be significantly different at the protein level, while FLT4 and MMP-2 were selected by the statistical classifier as key molecules in the separation of different prognostic groups. Figs 4-6 depict immunhistochemical slides stained with antibodies agains these ECM components. All 3 molecules exhibited increased expression in tumor samples from patients whose survival time was below average. These results supported previous data, further confirming their role in glioma invasion $(50,51)$. FLT4 is a receptor for VEGF-C and D, and it is not normally expressed in human brain endothelium; however, it has been previously determined to be overexpressed in the endothelium of blood vessels in glioblastoma (51). This expression may be responsible for the escape phenomenon of bevacizumab-treated patients, which is a secondary neovascularization despite anti-angiogenic therapy with bevacizumab, which primarily inhibits VEGF1 and VEGF2, and partially inhibits VEGF3 (50). MDM2 is an inhibitor of the p53 protein. An increased amount of MDM2 can explain a second way method to bypass the gatekeeper function of p53 in tumor protein 53 wild-type glioblastomas $(52,53)$. Furthermore, MDM2 has p53-independent roles. MDM2 may induce genomic instability through inhibiting DNA damage repair and suppressing cell cycle arrest (52). MDM2 has also been demonstrated to facilitate epithelial-mesenchymal transition; therefore, it enhances motility and tumor invasiveness (53). MMP2 has a well-described role in the dynamic alteration of tumor ECM, as well as other oncogenic functions that assist glioma cells with invading the brain parenchyma (54-57). All these molecules could serve as a target for future anti-invasive therapies in glioblastoma treatment.

In the present study, the statistical classifier nearest neighbor search was able to identify the prognostic group for each sample based upon the invasion spectrum, including the expressional pattern of the invasion-associated molecules. The method had high accuracy in determining whether the patient belonged to group A or B. This is important from a clinician's point of view, as it is one of the first methods that could provide information on individual patient survival. The increased positive predictive value for patients who have a worse prognosis is beneficial in identifying those patients that require extra attention and care, and whose treatment protocol should be intensified and/or complemented with targeted therapy as first-line treatment. Additional information on the molecular composition of tumor samples is provided; thus, selecting the correct anti-invasive therapeutic agent in the future would be possible. Therefore, the invasion spectrum can be considered as a prognostic factor with a future predictive role in patients with glioblastoma.

\section{Acknowledgements}

Not applicable.

\section{Funding}

The present study was supported by the Hungarian Brain Research Program (grant no 2017-1.2.1-NKP-2017-00002) and the ÚNKP-17-3-I and ÚNKP-17-2-I New National Excellence Program of the Ministry of Human Capacities.

\section{Availability of data and materials}

The datasets used and/or analyzed during the present study are available from the corresponding author on reasonable request.

\section{Authors' contributions}

JV took part in the design of the study, carried out IHC staining and evaluation, took part in the statistical analysis and drafted the manuscript. LSz carried out IHC staining and evaluation, prepared patient data for analysis, took part in the statistical analysis and contributed to the drafting of the manuscript, $\mathrm{TH}$ analysed tissue samples and validated IHC slides, MKC took part in the IHC slide evaluation, GZ performed qRT-PCR, LS performed nearest neighbor search and validated other statistical analyses performed by JV and LSz. JT was responsible for oncologic treatments and patient documentation, contributed to the interpretation of results from patient data analyses, LB and JRP collected and prepared tumor samples and collected patient data, KA took part in the design of the study, revised the manuscript for intellectual content and gave final approval of the manuscript.

\section{Ethics approval and consent to participate}

The study was approved by the Scientific and Research Ethics Committee of the Medical Research Council (ref. no. 51450-2/2015/EKU).

\section{Patient consent for publication}

All patients had signed an informed consent form for academic and research use of the resected tissues, including publication of data and findings.

\section{Competing interests}

The authors declare that they have no competing interests.

\section{References}

1. Grossman SA, Ye X, Piantadosi S, Desideri S, Nabors LB Rosenfeld M, Fisher J and NABTT CNS Consortium: Survival of patients with newly diagnosed glioblastoma treated with radiation and temozolomide in research studies in the United States. Clin Cancer Res 16: 2443-2449, 2010.

2. Back MF, Ang ELL, Ng WH, See SJ, Lim CCT, Chan SP and Yeo TT: Improved median survival for glioblastoma multiforme following introduction of adjuvant temozolomide chemotherapy. Ann Acad Med Singapore 36: 338-342, 2007.

3. Chamberlain MC: Bevacizumab for the treatment of recurrent glioblastoma. Clin Med Insights Oncol 5: 117-129, 2011.

4. Stupp R, Mason WP, van den Bent MJ, Weller M, Fisher B, Taphoorn MJ, Belanger K, Brandes AA, Marosi C, Bogdahn U, et al: Radiotherapy plus concomitant and adjuvant temozolomide for glioblastoma. N Engl J Med 352: 987-996, 2005. 
5. Krex D, Klink B, Hartmann C, von Deimling A, Pietsch T, Simon M, Sabel M, Steinbach JP, Heese O, Reifenberger G, et al: Long-term survival with gliossblastoma multiforme. Brain 130: 2596-606, 2007.

6. Donato V, Papaleo A, Castrichino A, Banelli E, Giangaspero F Salvati M and Delfini R: Prognostic implication of clinical and pathologic features in patients with glioblastoma multiforme treated with concomitant radiation plus temozolomide. Tumori 93: 248-256, 2007.

7. Sanai N and Berger MS: Glioma extent of resection and its impact on patient outcome. Neurosurgery 62: 753-764, 2008.

8. Griffin CA, Burger P, Morsberger L, Yonescu R, Swierczynski S, Weingart JD and Murphy KM: Identification of der(1;19)(q10;p10) in five oligodendrogliomas suggests mechanism of concurrent $1 p$ and 19q loss. J Neuropathol Exp Neurol 65: 988-994, 2006.

9. Reifenberger G, Hentschel B, Felsberg J, Schackert G, Simon M, Schnell O, Westphal M, Wick W, Pietsch T, Loeffler M, et al Predictive impact of MGMT promoter methylation in glioblastoma of the elderly. Int J Cancer 131: 1342-1350, 2012.

10. Louis DN, Perry A, Reifenberger G, von Deimling A, Figarella-Branger D, Cavenee WK, Ohgaki H, Wiestler OD, Kleihues P and Ellison DW: The 2016 world health organization classification of tumors of the central nervous system: A summary. Acta Neuropathol 113: 803-820, 2016

11. Bleeker FE, Molenaar RJ and Leenstra S: Recent advances in the molecular understanding of glioblastoma. J Neurooncol 108: $11-27,2012$.

12. Schwartzbaum JA, Fisher JL, Aldape KD and Wrensch M: Epidemiology and molecular pathology of glioma. Nat Clin Pract Neurol 2: 494-503, 2006

13. Demuth T and Berens ME: Molecular mechanisms of glioma cell migration and invasion. J Neurooncol 70: 217-228, 2004.

14. Milinkovic V, Bankovic J, Rakic M, Stankovic T, Skender-Gazibara M, Ruzdijic S and Tanic N: Identification of novel genetic alterations in samples of malignant glioma patients. PLoS One 8: e82108, 2013.

15. Cohen AL, Holmen SL and Colman H: IDH1 and IDH2 mutations in gliomas. Curr Neurol Neurosci Rep 13: 345, 2013.

16. Hartmann C, Hentschel B, Wick W, Capper D, Felsberg J, Simon M, Westphal M, Schackert G, Meyermann R and Pietsch T: Patients with IDH1 wild type anaplastic astrocytomas exhibit worse prognosis than IDH1-mutated glioblastomas, and IDH1 mutation status accounts for the unfavorable prognostic effect of higher age: Implications for classification of gliomas. Acta Neuropathol 120: 707-718, 2010.

17. Tysnes BB and Mahesparan R: Biological mechanisms of glioma invasion and potential therapeutic targets. J Neurooncol 53: 129-147, 2001.

18. Gladson CL: The extracellular matrix of gliomas: Modulation of cell function. J Neuropathol Exp Neurol 58: 1029-1040, 1999.

19. Reinhard J, Brösicke N, Theocharidis U and Faissner A: The extracellular matrix niche microenvironment of neural and cancer stem cells in the brain. Int J Biochem Cell Biol 81: 174-183, 2016.

20. Goldbrunner RH, Bernstein JJ and Tonn JC: Cell-extracellular matrix interaction in glioma invasion. Acta Neurochir (Wien) 141: 295-305, 1999.

21. Kreisl TN, McNeill KA, Sul J, Iwamoto FM, Shih J and Fine HA A phase I/II trial of vandetanib for patients with recurrent malignant glioma. Neuro Oncol 14: 1519-1526, 2012.

22. Razis E, Selviaridis P, Labropoulos S, Norris JL, Zhu MJ Song DD, Kalebic T, Torrens M, Kalogera-Fountzila A, Karkavelas G, et al: Phase II study of neoadjuvant imatinib in glioblastoma: Evaluation of clinical and molecular effects of the treatment. Clin Cancer Res 15: 6258-6266, 2009.

23. Shirai K, Siedow MR and Chakravarti A: Antiangiogenic therapy for patients with recurrent and newly diagnosed malignant gliomas. J Oncol 2012: 193436, 2012.

24. Virga J, Bognar L, Hortobagyi T, Zahuczky G, Csősz É, Kalló G, Tóth J, Hutóczki G, Reményi Puskár J, Steiner L and Klekner A: Prognostic role of the expression of invasion-related molecules in glioblastoma. J Neurol Surg A Cent Eur Neurosurg 78: 12-19, 2017.

25. Virga J, Bognár L, Hortobágyi T, Zahuczky G, Csősz É, Kalló G, Tóth J, Hutóczki G, Reményi Puskár J, Steiner L and Klekner A: Tumor grade vs. expression of invasion-related molecules in astrocytoma. Pathol Oncol Res 24: 35-43, 2018.

26. Virga J, Szemcsak CD, Remenyi-Puskar J, Tóth J, Hortobágyi T, Csốsz É, Zahuczky G, Szivos L, Bognár L and Klekner A Differences in extracellular matrix composition and its role in invasion in primary and secondary intracerebral malignancies. Anticancer Res 37: 4119-4126, 2017.
27. Mellinghoff IK, Wang MY, Vivanco I, Haas-Kogan DA, Zhu S, Dia EQ, Lu KV, Yoshimoto K, Huang JH, Chute DJ, et al: Molecular determinants of the response of glioblastomas to EGFR kinase inhibitors. N Engl J Med 353: 2012-2024, 2005.

28. Wild-Bode C, Weller M and Wick W: Molecular determinants of glioma cell migration and invasion. J Neurosurg 94: 978-984, 2001.

29. Bellail AC, Hunter SB, Brat DJ, Tan C and Van Meir EG: Microregional extracellular matrix heterogeneity in brain modulates glioma cell invasion. Int J Biochem Cell Biol 36: 1046-1069, 2004.

30. Gerber NK, Goenka A, Turcan S, Reyngold M, Makarov V, Kannan K, Beal K, Omuro A, Yamada Y, Gutin P, et al: Transcriptional diversity of long-term glioblastoma survivors. Neuro Oncol 16: 1186-1195, 2014.

31. Petrás M, Hutóczki G, Varga I, Vereb G, Szöllosi J, Bognár L, Ruszthi P, Kenyeres A, Tóth J, Hanzély Z, et al: Expression pattern of invasion-related molecules in cerebral tumors of different origin. Magy Onkológia 53: 253-258, 2009.

32. Varga I, Hutóczki G, Petrás M, Scholtz B, Mikó E, Kenyeres A, Tóth J, Zahuczky G, Bognár L, Hanzély Z and Klekner A: Expression of invasion-related extracellular matrix molecules in human glioblastoma vs. intracerebral lung adenocarcinoma metastasis. Cent Eur Neurosurg 71: 173-180, 2010.

33. Livak KJ and Schmittgen TD: Analysis of relative gene expression data using real-time quantitative PCR and the 2(-Delta Delta C(T)) Method. Methods 25: 402-408, 2001

34. Bondarenko A, Angrisani N, Meyer-Lindenberg A, Seitz JM, Waizy $\mathrm{H}$ and Reifenrath J: Magnesium-based bone implants: Immunohistochemical analysis of peri-implant osteogenesis by evaluation of osteopontin and osteocalcin expression. J Biomed Mater Res A 102: 1449-1457, 2014

35. Li L, Darden TA, Weinberg CR, Levine AJ and Pedersen LG: Gene assessment and sample classification for gene expression data using a genetic algorithm/k-nearest neighbor method. Comb Chem High Throughput Screen 4: 727-739, 2001

36. Manocha S and Girolami MA: An empirical analysis of the probabilistic K-nearest neighbour classifier. Pattern Recognit Lett 28: 1818-1824, 2007.

37. Birol Sarica F, Tufan K, Cekinmez M, Sen O, Cem Onal H, Mertsoylu H, Topkan E, Pehlivan B, Erdogan B and Nur Altinors M: Effectiveness of temozolomide treatment used at the same time with radiotherapy and adjuvant temozolomide; concomitant therapy of glioblastoma multiforme: Multivariate analysis and other prognostic factors. J Neurosurg Sci 54: 7-19, 2010

38. Helseth R, Helseth E, Johannesen TB, Langberg CW, Lote K, Rønning P, Scheie D, Vik A and Meling TR: Overall survival, prognostic factors, and repeated surgery in a consecutive series of 516 patients with glioblastoma multiforme. Acta Neurol Scand 122: 159-167, 2010

39. Chintala SK and Rao JK: Invasion of human glioma: Role of extracellular matrix proteins. Front Biosci 1: d324-d339, 1996.

40. Jung S, Moon KS, Kim ST, Ryu HH, Lee YH, Jeong YI, Jung TY, Kim IY, Kim KK and Kang SS: Increased expression of intracystic matrix metalloproteinases in brain tumors: relationship to the pathogenesis of brain tumor-associated cysts and peritumoral edema. J Clin Neurosci 14: 1192-1198, 2007.

41. Abdollahi A, Griggs DW, Zieher H, Roth A, Lipson KE, Saffrich R, Gröne HJ, Hallahan DE, Reisfeld RA, Debus J, et al: Inhibition of alpha(v)beta3 integrin survival signaling enhances antiangiogenic and antitumor effects of radiotherapy. Clin Cancer Res 11: 6270-6279, 2005.

42. Kawataki T, Yamane T, Naganuma H, Rousselle P, Andurén I, Tryggvason K and Patarroyo M: Laminin isoforms and their integrin receptors in glioma cell migration and invasiveness: Evidence for a role of alpha5-laminin(s) and alpha3beta1 integrin. Exp Cell Res 313: 3819-3831, 2007.

43. Yoshida T, Matsuda Y, Naito Z and Ishiwata T: CD44 in human glioma correlates with histopathological grade and cell migration. Pathol Int 62: 463-470, 2012.

44. Vuoriluoto K, Högnäs G, Meller P, Lehti K and Ivaska J Syndecan-1 and -4 differentially regulate oncogenic K-ras dependent cell invasion into collagen through $\alpha 2 \beta 1$ integrin and MT1-MMP. Matrix Biol 30: 207-217, 2011.

45. Aldape KD, Ballman K, Furth A, Buckner JC, Giannini C, Burger PC, Scheithauer BW, Jenkins RB and James CD: Immunohistochemical detection of EGFRvIII in high malignancy grade astrocytomas and evaluation of prognostic significance. J Neuropathol Exp Neurol 63: 700-707, 2004.

46. Holdhoff $M$ and Grossman SA: Controversies in the adjuvant therapy of high-grade gliomas. Oncologist 16: 351-358, 2011. 
47. Johnson DR and Galanis E: Medical management of high-grade astrocytoma: Current and emerging therapies. Semin Oncol 41: 511-522, 2014

48. Narita Y: Bevacizumab for glioblastoma. Ther Clin Risk Manag 11: 1759-1765, 2015.

49. Chinot OL, Wick W, Mason W, Henriksson R, Saran F, Nishikawa R, Carpentier AF, Hoang Xuan K, Kavan P, Cernea D, et al: Bevacizumab plus radiotherapy-temozolomide for newly diagnosed glioblastoma. N Engl J Med 370: 709-722, 2014.

50. Grau SJ, Trillsch F, Herms J, Thon N, Nelson PJ, Tonn JC and Goldbrunner R: Expression of VEGFR3 in glioma endothelium correlates with tumor grade. J Neurooncol 82: 141-150, 2007.

51. Jenny B, Harrison JA, Baetens D, Tille JC, Burkhardt K, Mottaz H, Kiss JZ, Dietrich PY, De Tribolet N, Pizzolato GP and Pepper MS: Expression and localization of VEGF-C and VEGFR-3 in glioblastomas and haemangioblastomas. J Pathol 209: 34-43, 2006.

52. Li Q and Lozano G: Molecular pathways: Targeting Mdm2 and Mdm4 in cancer therapy. Clin Cancer Res 19: 34-41, 2013.
53. Hientz K, Mohr A, Bhakta-Guha D and Efferth T: The role of p53 in cancer drug resistance and targeted chemotherapy. Oncotarget 8: 8921-8946, 2017.

54. Kesanakurti D, Chetty C, Dinh DH, Gujrati M and Rao JS: Role of MMP-2 in the regulation of IL-6/Stat3 survival signaling via interaction with $\alpha 5 \beta 1$ integrin in glioma. Oncogene 32: 327-340, 2013.

55. Deryugina EI, Bourdon MA, Luo GX, Reisfeld RA and Strongin A: Matrix metalloproteinase-2 activation modulates glioma cell migration. J Cell Sci 110: 2473-2482, 1997.

56. Deryugina EI, Bourdon MA, Reisfeld RA and Strongin A: Remodeling of collagen matrix by human tumor cells requires activation and cell surface association of matrix metalloproteinase-2. Cancer Res 58: 3743-3750, 1998.

57. Egeblad M and Werb Z: New functions for the matrix metalloproteinases in cancer progression. Nat Rev Cancer 2: 161-174, 2002.

(c) (i)

This work is licensed under a Creative Commons Attribution 4.0 International (CC BY 4.0) License. 